Journal of Applied AnALysis

Vol. 8, No. 2 (2002), pp. 245-259

\title{
NONLOCAL IN TIME PROBLEMS FOR EVOLUTION EQUATIONS OF SECOND ORDER
}

\author{
G. A. AVAlishVili \\ Received April 4, 2001 and, in revised form, January 3, 2002
}

\begin{abstract}
In this paper, nonlocal in time problem for abstract evolution equation of second order is studied and theorem on existence and uniqueness of its solution is proved. Some applications of this theorem for hyperbolic partial differential equations and systems are considered and it is proved, that well-posedness of the mentioned problems depends on algebraic properties of ratios between the dimensions of the spatial boundary and the times appearing in the nonlocal in time initial conditions.
\end{abstract}

\section{Introduction}

Nonlocal in time problems are non-classical initial boundary value problems, where instead of classical initial conditions we have a combination of the initial value of the solution and values of the solution for later times. These problems are generalizations of periodical problem, which is a particular case of problem of controllability by the initial conditions, where we seek for such initial conditions that state of the dynamical system at a certain moment of time coincides with its initial state.

2000 Mathematics Subject Classification. 11J68, 35L55, 35L90.

Key words and phrases. Second-order evolution equation, nonlocal in time conditions, algebraic numbers.

ISSN 1425-6908（C) Heldermann Verlag. 
Nonlocal in time problems were formulated and studied in [4] for parabolic equations. Later in [5-7] problems of this type were investigated for some equations of mathematical physics. In the present paper we study nonlocal in time problem for abstract evolution equation of second order and generalize results obtained before for hyperbolic equations in $[6,7]$. We investigate dependence of well-posedness of nonlocal in time problems on algebraic properties of expressions, which contain given moments of time and dimensions of the spatial boundary.

The outline of the paper is the following. In Section 2 the variational formulation of nonlocal in time problem for abstract evolution equation of second order is given and theorem on existence and uniqueness of its solution is proved. In Section 3 we consider some applications of the general theorem formulated in Section 2 for hyperbolic partial differential equations and systems. We show that for some nonlocal in time problems for hyperbolic equations in case of a parallelepiped as spatial domain, the existence and uniqueness of a solution depend on algebraic properties of ratios of the moments of time and lengths of the sides of the parallelepiped.

\section{An abstract nonlocal in time problem}

Let $V \subset H$ be separable Hilbert spaces such that the injection $V \hookrightarrow H$ is continuous and $V$ is dense in $H$. Denote with $(.,$.$) scalar product in H$. Assume that $H$ is identified with its dual space by scalar product in $H$, then it is possible to construct continuous and dense injection of $H$ in $V^{\prime}\left(V^{\prime}\right.$ is a dual space of $V)([2])$. Therefore we obtain

$$
V \hookrightarrow H \hookrightarrow V^{\prime}
$$

with continuous and dense injections. Let $\mathfrak{L}(X ; Y)$ denotes the space of linear continuous operators from $X$ to $Y\left(X, Y\right.$ are Banach spaces). $L^{p}(0, T ; X)$ denotes the space of measurable functions $g:(0, T) \rightarrow X$ equipped with the norm

$$
\begin{aligned}
& \|g\|_{L^{p}(0, T ; X)} \equiv\left(\int_{0}^{T}\|g(t)\|_{X}^{p} d t\right)^{1 / p}<+\infty, \quad \text { for } p<+\infty, \\
& \|g\|_{L^{p}(0, T ; X)} \equiv \sup _{t \in[0, T]} \operatorname{ess}\|g(t)\|_{X}<+\infty, \quad \text { for } p=+\infty \text {. }
\end{aligned}
$$

$C^{0}([0, T] ; X)$ is the space of continuous functions of $t \in[0, T]$ with values in $X$. In the case of $X=\mathbb{R}, C^{0}([0, T] ; X)$ denotes the space $C^{0}([0, T])$ of continuous real-valued functions on $[0, T]$. From the definition of Bochner's 
integral it follows that function $g \in L^{p}(0, T ; X)$ can be identified with distribution in $(0, T)$ with values in $X([9])$, and denote with

$$
g^{\prime}=\frac{d g}{d t} \in \mathfrak{D}^{\prime}((0, T) ; X)=\mathfrak{L}(\mathfrak{D}(0, T) ; X)
$$

the derivative of $g$ in the sense of distributions $(\mathfrak{D}(0, T)$ stands for the space of infinitely differentiable functions with compact support in $(0, T))$.

Furthermore, let $A \in \mathfrak{L}\left(V ; V^{\prime}\right)$ be a linear continuous operator such that the bilinear form $a(\varphi, \psi)=\langle A \varphi, \psi\rangle_{V^{\prime}, V}\left(\langle., .\rangle_{V^{\prime}, V}\right.$ denotes duality between $V$ and $\left.V^{\prime}\right)$ is symmetric and coercive, i.e.

$$
\begin{array}{ll}
a(\varphi, \psi)=a(\psi, \varphi), & \forall \varphi, \psi \in V, \\
a(\varphi, \varphi) \geq \alpha\|\varphi\|_{V}^{2}, \quad \alpha=\mathrm{const}>0, & \forall \varphi \in V,
\end{array}
$$

and the set of eigenvectors of the operator $A$ is complete in $V$.

The variational formulation of the nonlocal in time problem for abstract evolution equation of second order

$$
\frac{d^{2} u}{d t^{2}}+A u=f, \quad t \in(0, T)
$$

is as follows: find a function $u \in C^{0}([0, T] ; V), u^{\prime} \in C^{0}([0, T] ; H)$, which satisfies equation

$$
\frac{d}{d t}\left(u^{\prime}(.), v\right)+a(u(.), v)=(f(.), v), \quad \forall v \in V,
$$

in the sense of distributions in $(0, T)$ and the following nonlocal initial conditions

$$
\begin{aligned}
& u(0)=B u+u_{0}, \\
& u^{\prime}(0)=C u^{\prime}+u_{1},
\end{aligned}
$$

where $u_{0} \in V, u_{1} \in H, f \in L^{2}(0, T ; H)$,

$$
B \in \mathfrak{L}\left(C^{0}([0, T] ; V) ; V\right), \quad C \in \mathfrak{L}\left(C^{0}([0, T] ; H) ; H\right) .
$$

For the formulated problem we have the following

Theorem 2.1. Suppose that there exist linear continuous functionals $b, c$ : $C^{0}([0, T]) \rightarrow \mathbb{R}$ such that

$$
B\left(h(t) v_{n}\right)=b(h(t)) v_{n}, \quad C\left(h(t) v_{n}\right)=c(h(t)) v_{n}, \quad \forall h \in C^{0}([0, T]),
$$

for all $n \in \mathbb{N}$, where $\left\{v_{n}\right\}_{n \in \mathbb{N}}$ is an orthonormal (in $H$ ) system of eigenvectors of the operator $A$ with eigenvalues $\left\{\lambda_{n}^{2}\right\}_{n \in \mathbb{N}}$. If there exists a real positive number $q>0$ for which the inequality

$$
\begin{aligned}
& \left|\left(1-b\left(\cos \left(\lambda_{n} t\right)\right)\right)\left(1-c\left(\cos \left(\lambda_{n} t\right)\right)\right)+b\left(\sin \left(\lambda_{n} t\right)\right) c\left(\sin \left(\lambda_{n} t\right)\right)\right| \\
& \quad>q
\end{aligned}
$$


holds for all $n \in \mathbb{N}$, then nonlocal problem (2.2), (2.3) has a unique solution. Moreover, the mapping $\left\{f, u_{0}, u_{1}\right\} \rightarrow\{u, d u / d t\}$ is continuous from $L^{2}(0, T ; H) \times V \times H$ to $C^{0}([0, T] ; V) \times C^{0}([0, T] ; H)$.

Proof. Let us seek a solution of the formulated problem by the generalized Fourier series

$$
u(t)=\sum_{n=1}^{\infty} u_{n}(t) v_{n} .
$$

Inserting (2.5) into equation (2.2) and after some formal transformations, we obtain

$$
\begin{aligned}
u_{n}(t)= & \frac{1}{\lambda_{n}} \int_{0}^{t} f_{n}(\tau) \sin \left(\lambda_{n}(t-\tau)\right) d \tau \\
& +A_{n} \cos \left(\lambda_{n} t\right)+B_{n} \sin \left(\lambda_{n} t\right),
\end{aligned}
$$

where $f(t)=\sum_{n=1}^{\infty} f_{n}(t) v_{n}, f_{n}(t)=\left(f(t), v_{n}\right)$. Coefficients $A_{n}, B_{n}$ can be calculated from the conditions (2.3), i.e.

$$
\begin{aligned}
& \left.A_{n}\left(1-b\left(\cos \left(\lambda_{n} t\right)\right)\right)-B_{n} b\left(\sin \left(\lambda_{n} t\right)\right)\right) \\
& =u_{0 n}+\frac{1}{\lambda_{n}} b\left(\int_{0}^{t} f_{n}(\tau) \sin \left(\lambda_{n}(t-\tau)\right) d \tau\right), \\
& A_{n} c\left(\sin \left(\lambda_{n} t\right)\right)+B_{n}\left(1-c\left(\cos \left(\lambda_{n} t\right)\right)\right) \\
& =\frac{u_{1 n}}{\lambda_{n}}+\frac{1}{\lambda_{n}} c\left(\int_{0}^{t} f_{n}(\tau) \cos \left(\lambda_{n}(t-\tau)\right) d \tau\right),
\end{aligned}
$$

where $u_{0 n}=\left(u_{0}, v_{n}\right), u_{1 n}=\left(u_{1}, v_{n}\right)$. From (2.7), taking (2.4) into account, we can uniquely determine $A_{n}, B_{n}$ for which the following estimate is valid:

$$
\begin{aligned}
& \max \left(\left|A_{n}\right|,\left|B_{n}\right|\right) \leq C_{1}\left|u_{0 n}\right|+C_{2}\left|\frac{u_{1 n}}{\lambda_{n}}\right| \\
& +C_{3} \frac{1}{\left|\lambda_{n}\right|}\left(\int_{0}^{T} f_{n}^{2}(\tau) d \tau\right)^{1 / 2}(\|b\|+\|c\|),
\end{aligned}
$$

where $\|b\|,\|c\|$ are norms of the functionals $b, c$ respectively.

Let us consider series (2.5), where $u_{n}(t)$ is replaced by expression (2.6), and prove that it is a solution of the formulated nonlocal in time problem.

First, we show that series (2.5) converges uniformly with respect to $t$ in the space $V$. Denote with

$$
F_{n}(t)=\frac{1}{\lambda_{n}} \int_{0}^{t} f_{n}(\tau) \sin \left(\lambda_{n}(t-\tau)\right) d \tau \equiv \frac{\gamma_{n}(t)}{\lambda_{n}}
$$


and prove, that the series $\sum_{n=1}^{\infty} \gamma_{n}^{2}(t)$ converges uniformly with respect to $t$, and its sum is less than $T \int_{0}^{\bar{T}}\|f\|_{H}^{2} d t$. Indeed,

$$
\gamma_{n}^{2}(t) \leq \int_{0}^{t} \sin ^{2}\left(\lambda_{n}(t-\tau)\right) d \tau \int_{0}^{t} f_{n}^{2}(\tau) d \tau \leq T \int_{0}^{T} f_{n}^{2}(\tau) d \tau,
$$

i.e., the series $\sum_{n=1}^{\infty} \gamma_{n}^{2}(t)$ is dominated by the converging number series $T \sum_{n=1}^{\infty} \int_{0}^{T} f_{n}^{2}(\tau) d \tau$, the sum of which is equal to $T\|f\|_{L^{2}(0, T ; H)}^{2}$.

By the coerciveness of the bilinear form $a(.,$.$) , we obtain$

$$
\begin{aligned}
0 & \leq a\left(u_{0}-\sum_{n=1}^{N} \beta_{n} \frac{v_{n}}{\lambda_{n}}, u_{0}-\sum_{n=1}^{N} \beta_{n} \frac{v_{n}}{\lambda_{n}}\right)=a\left(u_{0}, u_{0}\right) \\
& -2 \sum_{n=1}^{N} \beta_{n} a\left(u_{0}, \frac{v_{n}}{\lambda_{n}}\right)+\sum_{n=1}^{N} \beta_{n}^{2} a\left(\frac{v_{n}}{\lambda_{n}}, \frac{v_{n}}{\lambda_{n}}\right) \\
& =a\left(u_{0}, u_{0}\right)-\sum_{n=1}^{N} \beta_{n}^{2}
\end{aligned}
$$

where $\beta_{n}=\left(1 / \lambda_{n}\right) a\left(u_{0}, v_{n}\right)$. From the latter inequality it follows that $\sum_{n=1}^{\infty} \beta_{n}^{2} \leq a\left(u_{0}, u_{0}\right)$.

Taking into account estimate (2.8), $\sum_{n=1}^{\infty} u_{1 n}^{2}=\left\|u_{1}\right\|_{H}^{2}$ and $u_{0 n}=\left(u_{0}, v_{n}\right)$ $=\left(1 / \lambda_{n}^{2}\right) a\left(u_{0}, v_{n}\right)$, we obtain that the series $\sum_{n=1}^{\infty}\left(\lambda_{n} u_{n}(t)\right)^{2}$ is dominated by the converging number series $\widetilde{C}_{4} \sum_{n=1}^{\infty}\left(\int_{0}^{T} f_{n}^{2}(\tau) d \tau+u_{1 n}^{2}+\beta_{n}^{2}\right)$ and the following estimate holds

$$
\sum_{n=1}^{\infty}\left(\lambda_{n} u_{n}(t)\right)^{2} \leq C_{4}\left(\|f\|_{L^{2}(0, T ; H)}^{2}+\left\|u_{1}\right\|_{H}^{2}+\left\|u_{0}\right\|_{V}^{2}\right) .
$$

Let us denote $u_{p q}=\sum_{n=p}^{q} u_{n}(t) v_{n}$. Since the system $\left\{v_{s}\right\}_{s \in \mathbb{N}}$ is orthogonal in $H$, we get that it is orthogonal with respect to the form $a(.,$.$) , i.e.,$ $a\left(v_{s}, v_{s^{\prime}}\right)=\lambda_{n}^{2}\left(v_{s}, v_{s^{\prime}}\right)=0, s \neq s^{\prime}, s, s^{\prime} \in \mathbb{N}$. Consequently, for each $\varepsilon>0$ there is a natural number $N(\varepsilon) \in \mathbb{N}$ such that

$$
a\left(u_{p q}, u_{p q}\right)=\sum_{n=p}^{q}\left(\lambda_{n} u_{n}(t)\right)^{2}<\varepsilon, \quad \text { for } p \geq N(\varepsilon), q \geq p .
$$

From conditions (2.1) we obtain

$$
\left\|u_{p q}\right\|_{V}^{2} \leq \frac{1}{\alpha} \sum_{n=p}^{q}\left(\lambda_{n} u_{n}(t)\right)^{2}<\frac{\varepsilon}{\alpha}, \quad \text { for } p \geq N(\varepsilon), q \geq p,
$$


that implies the uniform (with respect to $t$ ) convergence of series (2.5). Therefore $u \in C^{0}([0, T] ; V)$ and the following estimate is valid

$$
\|u(t)\|_{V}^{2} \leq C_{5}\left(\|f\|_{L^{2}(0, T ; H)}^{2}+\left\|u_{1}\right\|_{H}^{2}+\left\|u_{0}\right\|_{V}^{2}\right) .
$$

Similarly it can be checked that the series obtained by differentiation of series (2.5) converges uniformly with respect to $t$ in the space $H$ and consequently $u^{\prime} \in C^{0}([0, T] ; H)$. From the construction of $u(t)$ it is obvious that $u(t)$ satisfies initial conditions (2.3). Therefore, it suffices to prove that $u(t)$ satisfies equation (2.2).

Indeed, as $f \in L^{2}(0, T ; H), u_{n}(t)$ has second derivative for almost all $t \in(0, T)$, which is equal to $-\lambda_{n}^{2} u_{n}(t)+f_{n}(t)$ and is square integrable in $(0, T)$. Hence, for any function $\varphi \in \mathfrak{D}(0, T)$ and $v \in V$ we have

$$
-\int_{0}^{T} u_{n}^{\prime}(\tau)\left(v_{n}, v\right) \varphi^{\prime}(\tau) d \tau=\int_{0}^{T}\left(f_{n}(\tau)-\lambda_{n}^{2} u_{n}(\tau)\right)\left(v_{n}, v\right) \varphi(\tau) d \tau
$$

and

$$
\begin{aligned}
& -\int_{0}^{T} \sum_{n=1}^{N} u_{n}^{\prime}(\tau)\left(v_{n}, v\right) \varphi^{\prime}(\tau) d \tau+\int_{0}^{T} \sum_{n=1}^{N} \lambda_{n}^{2} u_{n}(\tau)\left(v_{n}, v\right) \varphi(\tau) d \tau \\
& =\int_{0}^{T} \sum_{n=1}^{N} f_{n}(\tau)\left(v_{n}, v\right) \varphi(\tau) d \tau .
\end{aligned}
$$

Letting $N$ tend to $\infty$ in (2.11), we obtain

$$
-\int_{0}^{T}\left(u^{\prime}(\tau), v\right) \varphi^{\prime}(\tau) d \tau+\int_{0}^{T} a(u(\tau), v) \varphi(\tau) d \tau=\int_{0}^{T}(f(\tau), v) \varphi(\tau) d \tau
$$

and, therefore, $u(t)$ satisfies equation (2.2) in the sense of distributions in $(0, T)$.

So $u(t)$ is a solution of nonlocal problem (2.2), (2.3) and, according to the inequalities $(2.8),(2.9),(2.10)$, we have

$$
\begin{gathered}
\|u\|_{C^{0}([0, T] ; V)}^{2} \leq C_{5}\left(\|f\|_{L^{2}(0, T ; H)}^{2}+\left\|u_{1}\right\|_{H}^{2}+\left\|u_{0}\right\|_{V}^{2}\right), \\
\left\|\frac{d u}{d t}\right\|_{C^{0}([0, T] ; H)}^{2} \leq C_{6}\left(\|f\|_{L^{2}(0, T ; H)}^{2}+\left\|u_{1}\right\|_{H}^{2}+\left\|u_{0}\right\|_{V}^{2}\right),
\end{gathered}
$$

that implies continuity of the mapping $\left\{f, u_{0}, u_{1}\right\} \rightarrow\{u, d u / d t\}$ if problem (2.2), (2.3) has a unique solution.

Let us prove that the formulated nonlocal problem has at most one solution. Indeed, suppose that there exist two solutions $u(t)$ and $v(t)$ of the problem. Then their difference $w(t)=u(t)-v(t)$ is a solution of the homogeneous nonlocal in time problem, i.e., for $f \equiv 0, u_{0}=0, u_{1}=0$. Furthermore, 
$w(t)$ is the solution of classical evolution problem for the equation (2.2) with initial conditions $w(0)$ and $w^{\prime}(0)$, which has a unique solution and

$$
w(t)=\sum_{n=1}^{\infty}\left(\widetilde{A}_{n} \cos \left(\lambda_{n} t\right)+\widetilde{B}_{n} \sin \left(\lambda_{n} t\right)\right) v_{n} .
$$

Since $w(t)$ satisfies homogeneous conditions $(2.3)$, then $\left(\widetilde{A}_{n}, \widetilde{B}_{n}\right)$ is a solution of homogeneous system (2.7). According to the conditions (2.4), the determinant of the system is different from zero and, therefore, $\widetilde{A}_{n}=\widetilde{B}_{n}=0$, $\forall n \in \mathbb{N}$ and $w(t) \equiv 0$. Thus, $u(t) \equiv v(t)$ and solution is unique.

Remark 2.1. From the proof of the uniqueness of solution for nonlocal in time problem (2.2), (2.3) it follows, that if in the formulation of Theorem 2.1 we have $q=0$, then problem $(2.2),(2.3)$ has at most one solution.

\section{Nonlocal in time problems for hyperbolic equations and systems}

In the present section we consider some applications of the Theorem 2.1 for the nonlocal in time problems for hyperbolic equations and systems.

Let $\Omega \subset \mathbb{R}^{n}$ be a bounded domain with Lipschitz boundary. Denote with $H^{s}(\Omega)=W^{2, s}(\Omega)$ the Sobolev space of order $s \in \mathbb{N}, H_{0}^{s}(\Omega)=W_{0}^{2, s}(\Omega)$ is the closure of the set $\mathfrak{D}(\Omega)$ of infinitely differentiable functions with compact support in $\Omega$, in the space $H^{s}(\Omega)$. Assume that $a_{i j}, \rho(i, j=\overline{1, n})$ are functions defined in $\Omega$ such that

$$
\begin{gathered}
a_{i j}, \rho \in L^{\infty}(\Omega), \rho(x) \geq 0, a_{i j}(x)=a_{j i}(x), i, j=\overline{1, n}, \\
\exists \alpha>0, \sum_{i, j=1}^{n} a_{i j}(x) \xi_{i} \xi_{j} \geq \alpha\left(\xi_{1}^{2}+\ldots+\xi_{n}^{2}\right), \quad \forall\left(\xi_{1}, \ldots, \xi_{n}\right) \in \mathbb{R}^{n},
\end{gathered}
$$

almost everywhere in $\Omega$. Let $V=H_{0}^{1}(\Omega), H=L^{2}(\Omega)$,

$$
A u=-\sum_{i, j=1}^{n} \frac{\partial}{\partial x_{i}}\left(a_{i j}(x) \frac{\partial u}{\partial x_{j}}\right)+\rho(x) u,
$$

and as the initial conditions (2.3) take

$$
\begin{aligned}
& u(x, 0)=\sum_{i=1}^{k} \alpha_{i}^{1} u\left(x, T_{i}\right)+\sum_{i, j=1}^{k} \int_{T_{i}}^{T_{j}} \rho_{i j}^{1}(\tau) u(x, \tau) d \tau+u_{0}(x), \\
& u_{t}(x, 0)=\sum_{i=1}^{k} \alpha_{i}^{2} u_{t}\left(x, T_{i}\right)+\sum_{i, j=1}^{k} \int_{T_{i}}^{T_{j}} \rho_{i j}^{2}(\tau) u_{t}(x, \tau) d \tau+u_{1}(x),
\end{aligned}
$$


where $x \in \Omega, \rho_{i j}^{1}, \rho_{i j}^{2}$ are measurable bounded real-valued functions, $\alpha_{i}^{1}, \alpha_{i}^{2}$ are real constants, $T_{i} \in(0, T](i, j=\overline{1, k})$. Note that under these conditions the bilinear form

$$
a(\varphi, \psi)=\sum_{i, j=1}^{n} \int_{\Omega} a_{i j} \frac{\partial \varphi}{\partial x_{j}} \frac{\partial \psi}{\partial x_{i}} d x+\int_{\Omega} \rho \varphi \psi d x, \quad \forall \varphi, \psi \in H_{0}^{1}(\Omega),
$$

defined by the operator $A$, fulfills conditions (2.1) and the set of eigenfunctions of the operator $A$ is complete in $H_{0}^{1}(\Omega)$. Hence, from Theorem 2.1 it immediately follows validity of the similar theorem in the case of nonlocal in time problem for multidimensional hyperbolic equation

$$
\frac{\partial^{2} u}{\partial t^{2}}+A u=f, \quad \text { in } Q=\Omega \times(0, T) .
$$

Theorem 3.1. If there exists a real constant $q>0$ such that

$$
\begin{aligned}
& \mid \prod_{r=1}^{2}\left(1-\sum_{i=1}^{k} \alpha_{i}^{r} \cos \left(\lambda_{n} T_{i}\right)-\sum_{i, j=1}^{k} \int_{T_{i}}^{T_{j}} \rho_{i j}^{r}(\tau) \cos \left(\lambda_{n} \tau\right) d \tau\right) \\
& +\prod_{r=1}^{2}\left(\sum_{i=1}^{k} \alpha_{i}^{r} \sin \left(\lambda_{n} T_{i}\right)+\sum_{i, j=1}^{k} \int_{T_{i}}^{T_{j}} \rho_{i j}^{r}(\tau) \sin \left(\lambda_{n} \tau\right) d \tau\right) \mid \\
& >q,
\end{aligned}
$$

for all $n \in \mathbb{N}, u_{0} \in H_{0}^{1}(\Omega), u_{1} \in L^{2}(\Omega), f \in L^{2}(Q)$, then nonlocal in time problem (3.2), (3.3) with homogeneous boundary conditions has a unique solution $u \in C^{0}\left([0, T] ; H_{0}^{1}(\Omega)\right), u^{\prime} \in C^{0}\left([0, T] ; L^{2}(\Omega)\right)$.

Corollary 3.1. If in nonlocal conditions $(3.2) \rho_{i j}^{1} \equiv \rho_{i j}^{2} \equiv 0$ and coefficients $\alpha_{i}^{1}, \alpha_{i}^{2}(i, j=\overline{1, k})$ satisfy the inequality

$$
\sum_{i=1}^{k}\left(\left|\alpha_{i}^{1}\right|+\left|\alpha_{i}^{2}\right|\right)<1
$$

then the nonlocal problem has a unique solution.

Proof. According to Theorem 3.1, it suffices to check validity of condition (3.4). From (3.5) we obtain 


$$
\begin{aligned}
& \left(1-\sum_{i=1}^{k} \alpha_{i}^{1} \cos \left(\lambda_{n} T_{i}\right)\right)\left(1-\sum_{i=1}^{k} \alpha_{i}^{2} \cos \left(\lambda_{n} T_{i}\right)\right) \\
& +\left(\sum_{i=1}^{k} \alpha_{i}^{1} \sin \left(\lambda_{n} T_{i}\right)\right)\left(\sum_{i=1}^{k} \alpha_{i}^{2} \sin \left(\lambda_{n} T_{i}\right)\right) \\
& \geq 1-\sum_{i=1}^{k}\left|\alpha_{i}^{1}\right|-\sum_{i=1}^{k}\left|\alpha_{i}^{2}\right|>0
\end{aligned}
$$

and, instead of $q$, we can take any positive real number less than $1-\sum_{i=1}^{k}\left(\left|\alpha_{i}^{1}\right|+\left|\alpha_{i}^{2}\right|\right)$.

Now, we consider some particular cases of the nonlocal in time problem for hyperbolic equation and show an essential difference between classical and nonlocal problems.

Let $\Omega=(0, l), A \equiv-\partial^{2} / \partial x^{2}, f \equiv 0$ and conditions (3.2) are of the following form:

$$
\begin{aligned}
& u(x, 0)=\sum_{i=1}^{k} \alpha_{i} u\left(x, T_{i}\right)+u_{0}(x), \\
& u_{t}(x, 0)=\sum_{i=1}^{k} \alpha_{i} u_{t}\left(x, T_{i}\right)+u_{1}(x),
\end{aligned}
$$

where $\alpha_{i} \neq 0$ is a real constant, $T_{i} \in(0, T](i=\overline{1, k})$.

Therefore we obtain nonlocal in time problem for the string oscillation equation, which, according to Theorem 3.1, has a unique solution if $\sum_{i=1}^{k}\left|\alpha_{i}\right|$ $<1$. In the case of $\sum_{i=1}^{k}\left|\alpha_{i}\right|=1$, from inequality (3.4) and Remark 2.1, we obtain that if among the points $\left\{T_{i}\right\}_{i=1}^{k}$ at least one is such that the ratio $T_{i} / l$ is irrational, then the nonlocal problem has at most one solution. It must be pointed out that if $k=1$, then condition of irrationality of the ratio $T_{1} / l$ is a necessary and sufficient condition for the uniqueness of solution of the nonlocal problem. Moreover, for the special case of $T_{1} / l$, we have the theorem of the existence of a solution.

Theorem 3.2. Suppose that $T_{1} / l$ is an irrational algebraic number of degree $r>1$. If $u_{0} \in H^{r}(\Omega), u_{1} \in H^{r-1}(\Omega), u_{0}, A u_{0}, \ldots$, $A^{[(r-1) / 2]} u_{0}, u_{1}, \ldots, A^{[r / 2]-1} u_{1} \in H_{0}^{1}(\Omega)$, then nonlocal in time problem (3.3), (3.6) with homogeneous boundary conditions has a unique solution for any $\alpha_{1} \in \mathbb{R}([y]$ denotes the integer part of the real number $y)$. 
Proof. According to Liouville's Theorem ([3]), since $T_{1} / l$ is an algebraic irrational number of degree $r>1$, there exists $c>0$ such that

$$
\left|\frac{T_{1}}{l}-\frac{p}{n}\right| \geq \frac{c}{n^{r}}, \quad \forall n, p \in \mathbb{N},
$$

and, hence,

$$
\left|n \frac{T_{1}}{l}-p\right| \geq \frac{c}{n^{r-1}}, \quad \forall n, p \in \mathbb{N} .
$$

From the proof of Theorem 2.1

$$
\begin{aligned}
& A_{n}=\frac{u_{0 n}\left(1-\alpha_{1} \cos \left(\frac{\pi n}{l} T_{1}\right)\right)+\frac{u_{1 n} l}{\pi n} \alpha_{1} \sin \left(\frac{\pi n}{l} T_{1}\right)}{\left(1+\alpha_{1}^{2}\right)-2 \alpha_{1} \cos \left(\frac{\pi n}{l} T_{1}\right)}, \\
& B_{n}=\frac{\frac{u_{1 n} l}{\pi n}\left(1-\alpha_{1} \cos \left(\frac{\pi n}{l} T_{1}\right)\right)-u_{0 n} \alpha_{1} \sin \left(\frac{\pi n}{l} T_{1}\right)}{\left(1+\alpha_{1}^{2}\right)-2 \alpha_{1} \cos \left(\frac{\pi n}{l} T_{1}\right)},
\end{aligned}
$$

where

$$
u_{0 n}=\sqrt{\frac{2}{l}} \int_{0}^{l} u_{0}(x) \sin \left(\frac{\pi n}{l} x\right) d x, u_{1 n}=\sqrt{\frac{2}{l}} \int_{0}^{l} u_{1}(x) \sin \left(\frac{\pi n}{l} x\right) d x .
$$

Hence, if $\left|\alpha_{1}\right| \neq 1$, then the nonlocal problem has a unique solution.

Now consider the case when $\left|\alpha_{1}\right|=1$. We have:

for $\alpha_{1}=1$,

$$
A_{n}=\frac{u_{0 n}}{2}+\frac{u_{1 n} l}{2 \pi n} \cot \left(\frac{\pi n T_{1}}{2 l}\right), B_{n}=\frac{u_{1 n} l}{2 \pi n}-\frac{u_{0 n}}{2} \cot \left(\frac{\pi n T_{1}}{2 l}\right) ;
$$

for $\alpha_{1}=-1$,

$$
A_{n}=\frac{u_{0 n}}{2}-\frac{u_{1 n} l}{2 \pi n} \tan \left(\frac{\pi n T_{1}}{2 l}\right), B_{n}=\frac{u_{1 n} l}{2 \pi n}+\frac{u_{0 n}}{2} \tan \left(\frac{\pi n T_{1}}{2 l}\right) .
$$

Denote with $m(x)=\min \{(2 / \pi) x, 2-(2 / \pi) x\}, 0 \leq x \leq \pi$. It is easily checked that for all $n \in \mathbb{N}$,

$$
\begin{aligned}
& \left|\sin \left(\frac{\pi n T_{1}}{2 l}\right)\right|=\left|\sin \left(\frac{\pi n T_{1}}{2 l}-\pi\left[\frac{n T_{1}}{2 l}\right]\right)\right|>m\left(\frac{\pi n T_{1}}{2 l}-\pi\left[\frac{n T_{1}}{2 l}\right]\right), \\
& \left|\cos \left(\frac{\pi n T_{1}}{2 l}\right)\right|=\left|\cos \left(\frac{\pi n T_{1}}{2 l}-\pi\left[\frac{n T_{1}}{2 l}\right]\right)\right|>1+2\left[\frac{n T_{1}}{2 l}\right]-\frac{n T_{1}}{l},
\end{aligned}
$$

whence

Therefore,

$$
\left|\cot \left(\frac{\pi n T_{1}}{2 l}\right)\right| \leq \widetilde{c} n^{r-1},\left|\tan \left(\frac{\pi n T_{1}}{2 l}\right)\right| \leq \widetilde{c} n^{r-1} .
$$

$$
\max \left\{\left|A_{n}\right|,\left|B_{n}\right|\right\} \leq C_{1}\left(\left|u_{0 n}\right|+\frac{\left|u_{1 n}\right|}{n}\right) n^{r-1} .
$$


Since $A\left(\sin \left(\lambda_{n} x\right)\right)=\lambda_{n}^{2} \sin \left(\lambda_{n} x\right), \lambda_{n}=\pi n / l, n \in \mathbb{N}$ and $u_{0}, A u_{0}, \ldots$, $A^{[(r-1) / 2]} u_{0} \in H_{0}^{1}(\Omega)$, we obtain:

if $r$ is even $\left(r=2 r_{0}\right)$, then

$$
\begin{aligned}
u_{0 n} & =\sqrt{\frac{2}{l}} \frac{1}{\lambda_{n}^{2}} \int_{0}^{l} u_{0}(x) A\left(\sin \left(\lambda_{n} x\right)\right) d x=\sqrt{\frac{2}{l}} \frac{1}{\lambda_{n}^{2}} \int_{0}^{l}\left(A u_{0}\right)(x) \sin \left(\lambda_{n} x\right) d x \\
& =\ldots=\sqrt{\frac{2}{l}} \frac{1}{\lambda_{n}^{2 r_{0}}} \int_{0}^{l}\left(A^{r_{0}} u_{0}\right)(x) \sin \left(\lambda_{n} x\right) d x=\frac{\bar{u}_{0 n}}{\lambda_{n}^{2 r_{0}}},
\end{aligned}
$$

if $r$ is odd $\left(r=2 r_{0}+1\right)$, then

$$
\begin{aligned}
u_{0 n} & =\sqrt{\frac{2}{l}} \frac{1}{\lambda_{n}^{2 r_{0}}} \int_{0}^{l}\left(A^{r_{0}} u_{0}\right)(x) \sin \left(\lambda_{n} x\right) d x \\
& =\sqrt{\frac{2}{l}} \frac{1}{\lambda_{n}^{2 r_{0}+1}} \int_{0}^{l} \frac{d}{d x}\left(\left(A^{r_{0}} u_{0}\right)(x)\right) \frac{d}{d x}\left(\frac{1}{\lambda_{n}} \sin \left(\lambda_{n} x\right)\right) d x=\frac{\widetilde{u}_{0 n}}{\lambda_{n}^{2 r_{0}+1}},
\end{aligned}
$$

where $\sum_{n=1}^{\infty}\left(\bar{u}_{0 n}\right)^{2} \leq c_{2}\left\|u_{0}\right\|_{H^{r}(\Omega)}^{2}$,

$$
\begin{aligned}
0 & \leq \int_{0}^{l}\left(\frac{d}{d x}\left(\left(A^{r_{0}} u_{0}\right)(x)-\sum_{n=1}^{N} \frac{\widetilde{u}_{0 n}}{\lambda_{n}} \sqrt{\frac{2}{l}} \sin \left(\lambda_{n} x\right)\right)\right)^{2} d x \\
& =\int_{0}^{l}\left(\frac{d}{d x}\left(\left(A^{r_{0}} u_{0}\right)(x)\right)\right)^{2} d x-\sum_{n=1}^{N}\left(\widetilde{u}_{0 n}\right)^{2}
\end{aligned}
$$

whence $\sum_{n=1}^{\infty}\left(\widetilde{u}_{0 n}\right)^{2} \leq c_{3}\left\|u_{0}\right\|_{H^{r}(\Omega)}^{2}$. Thus,

$$
u_{0 n}=\frac{\widehat{u}_{0 n} l^{r}}{\pi^{r} n^{r}}, \quad \sum_{n=1}^{\infty}\left(\widehat{u}_{0 n}\right)^{2} \leq \widehat{c}\left\|u_{0}\right\|_{H^{r}(\Omega)}^{2},
$$

and similarly

$$
u_{1 n}=\frac{\widehat{u}_{1 n} l^{r-1}}{\pi^{r-1} n^{r-1}}, \quad \sum_{n=1}^{\infty}\left(\widehat{u}_{1 n}\right)^{2} \leq \widehat{c}\left\|u_{1}\right\|_{H^{r-1}(\Omega)}^{2} .
$$

From the latter estimates for $u_{0 n}, u_{1 n}$ we deduce, that the series

$$
u(x, t)=\sum_{n=1}^{\infty} \sqrt{\frac{2}{l}}\left(A_{n} \cos \left(\frac{\pi n}{l} t\right)+B_{n} \sin \left(\frac{\pi n}{l} t\right)\right) \sin \left(\frac{\pi n}{l} x\right)
$$

converges uniformly (with respect to $t$ ) in the space $H^{1}(\Omega)$ and the series obtained by differentiation of (3.7) converges uniformly in $L^{2}(\Omega)$. Thus $u \in C^{0}\left([0, T] ; H_{0}^{1}(\Omega)\right)$ and $u^{\prime} \in C^{0}\left([0, T] ; L^{2}(\Omega)\right)$.

Note, that similar results in the case of Dirichlet problem for hyperbolic equations are obtained in $[1,8]$. More precisely, a solution of the Dirichlet 
boundary value problem for the string oscillation equation is uniquely determined if and only if the ratio $\xi=T / l$ of the sides of the rectangle $[0, l] \times[0, T]$ is irrational; the solution exists for all boundary values, which are differentiable sufficiently many times, if $\xi$ cannot "too rapidly" be approximated by rationals.

Similarly we may consider two-dimensional and multidimensional nonlocal in time problem (3.3), (3.6) with homogeneous boundary conditions. Particularly, let $\Omega=\left(0, l_{1}\right) \times \ldots \times\left(0, l_{s}\right), A \equiv-\sum_{i=1}^{s} \partial^{2} / \partial x_{i}^{2}, f \equiv 0$. Before formulation of the analogue to Theorem 3.2 let us introduce the following definition.

Definition 3.1. Let $D_{s}=\left\{d_{n_{1} n_{2} \ldots n_{s}} ; n_{1}, n_{2}, \ldots, n_{s} \in \mathbb{N}_{0}=\mathbb{N} \cup\{0\}\right\}$ be an infinite set of non-negative real numbers. We say that the rate of approximation of the real number $d$ on the set $D_{s}$ is less than $r, r \in \mathbb{N}$, if there exists a constant $c>0$ such that for all $n_{1}, n_{2}, \ldots, n_{s} \in \mathbb{N}_{0}$,

$$
\left|d-\frac{p}{d_{n_{1} n_{2} \ldots n_{s}}}\right| \geq \frac{c}{d_{n_{1} n_{2} \ldots n_{s}}^{r}}, \quad \forall p \in \mathbb{N}, d_{n_{1} n_{2} \ldots n_{s}} \in D_{s}
$$

In the case of multidimensional nonlocal in time problem, if we have $\sum_{i=1}^{k}\left|\alpha_{i}\right|<1$, then problem (3.3), (3.6) has a unique solution. In the case of $\sum_{i=1}^{k}\left|\alpha_{i}\right|=1$, the nonlocal in time problem has at most one solution if there exists $T_{i}$ for which the equation

$$
\left(\frac{n_{1}}{l_{1}}\right)^{2}+\left(\frac{n_{2}}{l_{2}}\right)^{2}+\ldots+\left(\frac{n_{s}}{l_{s}}\right)^{2}=\left(\frac{p}{T_{i}}\right)^{2}
$$

is unsolvable in integers. Let us consider the case of $l_{1}=l_{2}=\ldots=l_{s}=l$ and $k=1$. According to the equation (3.8), the uniqueness of a solution of the nonlocal in time problem depends on algebraic properties of $T_{1} / l$. More precisely, the following theorem is valid.

Theorem 3.3. If $T_{1} / l$ is such, that $n_{1}, \ldots, n_{s}, p l / T_{1}$ are not the generalized Pythagorean numbers, i.e., don't satisfy equation (3.8) for any integers $n_{1}, \ldots, n_{s}, p$ and the rate of approximation of $T_{1} / l$ on the set $D_{s}=\left\{d_{n_{1} \ldots n_{s}}=\right.$ $\left.\sqrt{n_{1}^{2}+\ldots+n_{s}^{2}} ; n_{1}, \ldots, n_{s} \in \mathbb{N}_{0}\right\}$ is less than $r>1, u_{0} \in H^{r}(\Omega), u_{1} \in$ $H^{r-1}(\Omega), A^{k_{\gamma}} u_{\gamma} \in H_{0}^{1}(\Omega), 0 \leq k_{\gamma} \leq[(r-1+\gamma) / 2]-\gamma, \gamma=0,1$, then nonlocal problem (3.3), (3.6) with homogeneous boundary conditions has a unique solution for any $\alpha_{1} \in \mathbb{R}$.

Proof. We only sketch the proof, because it is similar to the proof of Theorem 3.2. Note, that if $\left|\alpha_{1}\right| \neq 1$, then the formulated theorem follows from 
Theorem 3.1. Let us consider the case of $\left|\alpha_{1}\right|=1$. As in the proof of Theorem 2.1 solution of the nonlocal problem we seek by Fourier series

$$
\begin{aligned}
& u\left(x_{1}, \ldots, x_{s}, t\right)=\sum_{n_{1}=1}^{\infty} \ldots \sum_{n_{s}=1}^{\infty}\left(A_{n_{1} \ldots n_{s}} \cos \left(\lambda_{n_{1} \ldots n_{s}} t\right)\right. \\
& \left.+B_{n_{1} \ldots n_{s}} \sin \left(\lambda_{n_{1} \ldots n_{s}} t\right)\right) v_{n_{1} \ldots n_{s}}\left(x_{1}, \ldots, x_{s}\right),
\end{aligned}
$$

where

$$
\begin{aligned}
& v_{n_{1} \ldots n_{s}}\left(x_{1}, \ldots, x_{s}\right)=\left(\frac{2}{l}\right)^{s / 2} \sin \left(\frac{\pi n_{1}}{l} x_{1}\right) \ldots \sin \left(\frac{\pi n_{s}}{l} x_{s}\right), n_{1}, \ldots, n_{s} \in \mathbb{N} . \\
& \lambda_{n_{1} \ldots n_{s}}=\frac{\pi \sqrt{n_{1}^{2}+\ldots+n_{s}^{2}}}{l},
\end{aligned}
$$

Coefficients $A_{n_{1} \ldots n_{s}}, B_{n_{1} \ldots n_{s}}$ determined from initial conditions (3.6), are of the following form:

$$
\begin{aligned}
& A_{n_{1} \ldots n_{s}}=\frac{u_{0 n_{1} \ldots n_{s}}}{2}+\frac{u_{1 n_{1} \ldots n_{s}}}{2 \lambda_{n_{1} \ldots n_{s}}}\left(\frac{\alpha_{1}+1}{2} \cot +\frac{\alpha_{1}-1}{2} \tan \right)\left(\frac{\lambda_{n_{1} \ldots n_{s}} T_{1}}{2}\right), \\
& B_{n_{1} \ldots n_{s}}=\frac{u_{1 n_{1} \ldots n_{s}}}{2 \lambda_{n_{1} \ldots n_{s}}}-\frac{u_{0 n_{1} \ldots n_{s}}}{2}\left(\frac{\alpha_{1}+1}{2} \cot +\frac{\alpha_{1}-1}{2} \tan \right)\left(\frac{\lambda_{n_{1} \ldots n_{s}} T_{1}}{2}\right) .
\end{aligned}
$$

Taking into account property of $T_{1} / l$, we obtain

$$
\begin{aligned}
& \max \left\{\left|A_{n_{1} \ldots n_{s}}\right|,\left|B_{n_{1} \ldots n_{s}}\right|\right\} \\
& \leq C\left(\left|u_{0 n_{1} \ldots n_{s}}\right|+\frac{u_{1 n_{1} \ldots n_{s}}}{{\sqrt{n_{1}^{2}+\ldots+n_{s}^{2}}}^{2}}\right){\sqrt{n_{1}^{2}+\ldots+n_{s}^{2}}}^{r-1},
\end{aligned}
$$

and from the latter estimate, as in the proof of Theorem 3.2, we deduce, that the function $u\left(x_{1}, \ldots, x_{s}, t\right)$ defined by series $(3.9)$ is a solution of the nonlocal in time problem (3.3), (3.6).

It must be pointed out that Theorem 2.1 allows to investigate nonlocal in time problem for hyperbolic system. Let $\Omega \subset \mathbb{R}^{n}$ be a bounded domain with regular boundary, $V=\mathfrak{H}_{0}^{m}(\Omega)=\left[H_{0}^{m}(\Omega)\right]^{N}, H=\mathfrak{L}^{2}(\Omega)=\left[L^{2}(\Omega)\right]^{N}$ and let $A$ be an elliptic operator of order $2 m$ :

$$
A=\sum_{k=0}^{m}(-1)^{k} \sum \frac{\partial^{k}}{\partial x_{i_{1}} \ldots \partial x_{i_{k}}}\left(A_{j_{1} \ldots j_{k}}^{i_{1} \ldots i_{k}}(x) \frac{\partial^{k}}{\partial x_{j_{1}} \ldots \partial x_{j_{k}}}\right),
$$

where in the inner sum each index $i_{1}, \ldots, i_{k}, j_{1}, \ldots, j_{k}$ independently range over the set $\{1, \ldots, n\} . u$ is $N$-component vector-function, $A_{j_{1} \ldots j_{k}}^{i_{1} \ldots i_{k}}(x)-$ square matrix of order $N$, which does not change for any transposition of upper or lower indices and turns into transpose of the matrix if all upper 
indices are transposed with all lower indices. Furthermore, assume that the elements of the matrices belong to $L^{\infty}(\Omega)$ and for almost all $x \in \Omega$,

$$
\begin{aligned}
& \left(\left(\sum A_{j_{1} \ldots j_{m}}^{i_{1} \ldots i_{m}}(x) t_{j_{1} \ldots j_{m}}, t_{i_{1} \ldots i_{m}}\right)\right) \geq \alpha \sum\left\|t_{i_{1} \ldots i_{m}}\right\|^{2}, \alpha>0, \\
& \left(\left(\sum A_{j_{1} \ldots j_{k}}^{i_{1} \ldots i_{k}}(x) t_{j_{1} \ldots j_{k}}, t_{i_{1} \ldots i_{k}}\right)\right) \geq 0, k=0,1, \ldots, m-1 .
\end{aligned}
$$

In (3.11) $t_{i_{1} \ldots i_{k}}$ is $N$-component vector, which does not change for any transposition of indices $i_{1}, \ldots, i_{k}(k=\overline{1, m}),((.,)),.\|\cdot\|$ denote the scalar product and norm in the $N$-dimensional Euclidean space, respectively.

Let us consider now nonlocal in time problem for hyperbolic system

$$
\frac{\partial^{2} u}{\partial t^{2}}+A u=f, \quad \text { in } Q=\Omega \times(0, T),
$$

with nonlocal initial conditions of the form (3.2) and homogeneous boundary conditions

$$
u=\frac{\partial u}{\partial \nu}=\ldots=\frac{\partial^{m-1} u}{\partial \nu^{m-1}}=0, \quad \text { on } \Gamma=\partial \Omega \times[0, T],
$$

where $\nu$ is the unit outward normal to $\partial \Omega$. In this case bilinear form $a(.,$. defined by the operator $A$ is of the following form

$$
a(u, v)=\int_{\Omega} \sum_{k=0}^{m} \sum\left(\left(A_{j_{1} \ldots j_{k}}^{i_{1} \ldots i_{k}}(x) \frac{\partial^{k} u}{\partial x_{j_{1}} \ldots \partial x_{j_{k}}}, \frac{\partial^{k} v}{\partial x_{i_{1}} \ldots \partial x_{i_{k}}}\right)\right) d x
$$

Taking into account conditions (3.11) it is not difficult to check that bilinear form $a(.,$.$) is symmetric and coercive on V \times V$. Moreover, it is well known, that $\mathfrak{H}_{0}^{m}(\Omega)$ is dense in $\mathfrak{L}^{2}(\Omega)$ and the set of eigenfunctions of the operator $A$ is complete in $V$.

So, applying Theorem 2.1, we obtain the following statement.

Theorem 3.4. If $u_{0} \in \mathfrak{H}_{0}^{m}(\Omega), u_{1} \in \mathfrak{L}^{2}(\Omega), f \in \mathfrak{L}^{2}(Q)$ and condition (3.4) is fulfilled, then the formulated nonlocal in time problem for the hyperbolic system has a unique solution $u \in C^{0}\left([0, T] ; \mathfrak{H}_{0}^{m}(\Omega)\right), u^{\prime} \in C^{0}\left([0, T] ; \mathfrak{L}^{2}(\Omega)\right)$.

Acknowledgement. The author is very grateful to Prof. David Gordeziani for useful discussions. 


\section{References}

[1] Bourgin, D. G., Duffin, R., The Dirichlet problem for the vibrating string equation, Bull. Amer. Math. Soc. 45 (1939), 851-859.

[2] Brezis, H., Analyse Fonctionelle, Masson, Paris, 1983.

[3] Gelfond, A. O., Transcendental and Algebraic Numbers (in Russian), Gosudarstv. Izdat. Tehn.-Teor. Lit., Moscow, 1952.

[4] Gordeziani, D. G., On some initial conditions for parabolic equations, Reports of the Enlarged Session of the Seminar of I. Vekua Institute of Applied Mathematics 4 (1989), 57-60.

[5] Gordeziani, D. G., On one problem for the Navier-Stokes equation, Abstracts, Contin. Mech. Related Probl. Anal., Tbilisi (1991), 83.

[6] Gordeziani, D.G., On solution of in time nonlocal problems for some equations of mathematical physics, ICM-94, Abstracts, Short Comm. (1994), 240.

[7] Gordeziani, D. G., Grigalashvili, Z., Non-local problems in time for some equations of mathematical physics, Dokl. Semin. Inst. Prikl. Mat. im. I. N. Vekua 22 (1993), 108-114.

[8] John, F., The Dirichlet problem for a hyperbolic equation, Amer. J. Math. 63 (1941), 141-154.

[9] Schwartz, L., Théorie des distributions à valeurs vectorielles (I), Ann. Inst. Fourier 7 (1957), 1-142.

Gia A. Avalishvili

Institute of ApPliEd Mathematics

TBILISI StATE UNIVERSity

UNIVERSITY STR. 2

380043 TBILISI

Georgia

EMAIL: GAVALISH@VIAM.HEPI.EDU.GE 